Research, Society and Development, v. 9, n. 9, e260996114, 2020

(CC BY 4.0) | ISSN 2525-3409 | DOI: http://dx.doi.org/10.33448/rsd-v9i9.6114

Análise bibliométrica de publicações sobre concreto com reaproveitamento de resíduos a partir da base de dados Web of Science

Bibliometric analysis of publications on concrete with waste reuse from the Web of

\title{
Science database
}

Análisis bibliométrico de publicaciones sobre concreto con reutilización de residuos de la base de datos de Web of Science

Recebido: 28/06/2020 | Revisado: 13/07/2020 | Aceito: 12/08/2020 | Publicado: 17/08/2020

Felipe José Marques Mesquita

ORCID: https://orcid.org/0000-0002-1589-1868

Universidade Federal do Pará, Brasil

E-mail: felipejmesquita@gmail.com

Taiana da Silva Ferreira

ORCID: https://orcid.org/0000-0003-4910-3767

Universidade Federal do Pará, Brasil

E-mail: taianadsf@gmail.com

Geilma Lima Vieira

ORCID: https://orcid.org/0000-0001-6148-3307

Universidade Federal do Espírito Santo, Brasil

E-mail: geilma.vieira@gmail.com

Heleno Fülber

ORCID: https://orcid.org/0000-0001-7836-593X

Universidade Federal do Pará, Brasil

E-mail: fulber@ufpa.br

Júnior Hiroyuki Ishihara

ORCID: https://orcid.org/0000-0002-0081-7913

Universidade Federal do Pará, Brasil

E-mail: hiroyuki_jr@hotmail.com

\section{Resumo}

A crescente demanda por materiais alternativos em substituição aos agregados naturais tem possibilitado o reaproveitamento de resíduos da construção civil para produção de concretos. Este aumento da busca por materiais alternativos só se tornou possível, além de outros fatores, 
por conta do aumento de publicações indexadas dentro dessa temática. O objetivo desta pesquisa foi realizar o levantamento bibliométrico das publicações sobre concreto com reaproveitamento de resíduos, permitindo a análise quantitativa e qualitativa sobre a evolução das publicações, os principais periódicos e eventos da área, os autores e instituições mais relevantes e os resíduos estudados. O trabalho aborda aspectos de publicações relacionadas ao tema de reaproveitamento de resíduos em concretos, utilizando ferramentas bibliométricas que permitiram analisar publicações brasileiras sobre o tema. $\mathrm{O}$ levantamento bibliométrico foi realizado durante o período de 1998 a 2017 na base de dados Web of Science e possibilitou a verificação dos autores e instituições mais produtivos, resíduos mais estudados, redes de cooperação entre autores e países, além dos periódicos e eventos mais influentes e significativos sobre o tema. $\mathrm{O}$ estudo revelou crescente interesse por este tema, com destaque ao resíduo de construção e demolição. Apesar da construção civil ser o tema de muitos trabalhos, as publicações brasileiras sobre concreto com reaproveitamento de resíduos ainda são incipientes, foram verificadas lacunas de publicações, como o baixo grau de colaboração entre autores da área, principalmente no âmbito internacional, com autores da Ásia e África, além da baixa frequência de publicações de trabalhos que utilizem resíduos na produção de concretos especiais.

Palavras-chave: Concreto; Resíduos; Bibliometria; Agregados reciclados; Sustentabilidade.

\section{Abstract}

The growing demand for alternative materials to replace natural aggregates has made it possible to reuse construction waste for the production of concrete. This increase in the search for alternative materials was only possible, in addition to other factors, due to the increase in publications indexed within this theme. The objective of this research was to carry out a bibliometric survey of publications on concrete with reuse of residues, allowing quantitative and qualitative analysis on the evolution of publications, the main journals and events in the area, the most relevant authors and institutions and the studied residues. The work addresses aspects of publications related to the topic of reuse of waste in concrete, using bibliometric tools that allowed analyzing Brazilian publications on the subject. The bibliometric survey was carried out from 1998 to 2017 in the Web of Science database and made it possible to verify the most productive authors and institutions, the most studied residues, cooperation networks between authors and countries, in addition to the most influential journals and events and significant on the topic. The study revealed growing interest in this topic, with emphasis on construction and demolition waste. Although civil construction is the subject of 
many works, Brazilian publications on concrete with reuse of residues are still incipient, there were gaps in publications, such as the low degree of collaboration between authors in the area, especially at the international level, with authors from Asia. and Africa, in addition to the low frequency of publication of works that use waste in the production of special concretes.

Keywords: Concrete; Waste; Bibliometry; Recycled aggregates; Sustainability.

\section{Resumen}

La creciente demanda de materiales alternativos que sustituyan a los áridos naturales ha permitido reutilizar los residuos de la construcción para la producción de hormigón. Este incremento en la búsqueda de materiales alternativos solo fue posible, además de otros factores, debido al incremento de publicaciones indexadas dentro de esta temática. El objetivo de esta investigación fue realizar el relevamiento bibliométrico de publicaciones sobre hormigón con reutilización de residuos, permitiendo analizar cuantitativamente y cualitativamente la evolución de las publicaciones, las principales revistas y eventos del área, los autores e instituciones más relevantes y los residuos estudiados. El trabajo aborda aspectos de las publicaciones relacionadas con el tema de la reutilización de residuos en el hormigón, utilizando herramientas bibliométricas que permitieron analizar las publicaciones brasileñas sobre el tema. La encuesta bibliométrica se realizó de 1998 a 2017 en la base de datos de Web of Science y permitió verificar los autores e instituciones más productivos, los residuos más estudiados, las redes de cooperación entre autores y países, además de las revistas y eventos más influyentes y significativo sobre el tema. El estudio reveló un creciente interés en este tema, con énfasis en los residuos de construcción y demolición. Si bien la construcción civil es objeto de muchos trabajos, las publicaciones brasileñas sobre concreto con reutilización de residuos aún son incipientes, existían vacíos en las publicaciones, como el bajo grado de colaboración entre autores del área, especialmente a nivel internacional, con autores de Asia. y África, además de la baja frecuencia de publicación de trabajos que utilizan residuos en la producción de hormigones especiales.

Palabras clave: Hormigón; Residuos; Bibliometría; Áridos reciclados; Sustentabilidad.

\section{Introdução}

O desenvolvimento econômico-populacional proporcionou o aumento na geração de resíduos nas cidades, criando a necessidade de áreas cada vez maiores para o descarte 
adequado destes resíduos. A disposição inadequada de resíduos pode ocasionar problemas ambientais como a contaminação do solo, água e ar, além da proliferação de vetores de doenças.

Conforme ABRELPE (Associação Brasileira de Empresas de Limpeza Pública e Resíduos Especiais) (2017), o Brasil produz aproximadamente 78,4 milhões de toneladas de resíduos sólidos urbanos anualmente.

O elevado consumo de materiais naturais torna a construção civil um potencial agente reciclador de resíduos (Moura et al., 2013). Estima-se que cerca de 50\% dos recursos naturais extraídos da natureza são utilizados na construção civil (Brasileiro; Matos, 2015). A incorporação de subprodutos de indústrias na produção de concreto ao substituir o material aglomerante ou os agregados contribui para conservação dos recursos naturais e melhora sua resistência e durabilidade (Moura et al., 2013).

A utilização de agregados reciclados no concreto tem sido tema de interesse de muitos pesquisadores, além do papel econômico, a utilização de agregados reciclados pode influenciar nas características do concreto no estado fresco e endurecido (Silva, 2006). Estudos como de Morais et al. (2020) que utilizou Resíduo de Cerâmica Vermelha e obteve bons resultados, são cada vez mais crescentes.

O fomento do conceito de sustentabilidade em setores produtivos tradicionais como a construção civil exige que certos paradigmas do desenvolvimento sejam quebrados e o fator ambiental seja contemplado desde o início do planejamento da produção. A busca de alternativas que beneficiem os anseios tanto dos produtores, que querem manter a qualidade de seus produtos, quanto da sociedade que deseja uma solução para a degradação contínua dos recursos naturais é um desafio que o meio acadêmico deve tomar para si (Cabral, 2007).

Existe uma escassez nos estudos voltados a análise das publicações científicas brasileiras na área de construção e tecnologia de construção, sendo desejável seu incentivo para a superação das carências e limitações do setor, além de contribuir para a melhor compreensão das especificidades de seus processos de desenvolvimento cientifico, econômico e tecnológico. (Soares et al., 2016).

O estudo bibliométrico permite mapear as áreas com déficit de publicações, permitindo aos pesquisadores focarem os esforços nestas lacunas, possibilitando o crescimento das publicações nacionais.

Neste sentido, o presente estudo objetiva realizar o levantamento bibliométrico das publicações sobre concreto com reaproveitamento de resíduos, permitindo a análise quantitativa e qualitativa sobre a evolução das publicações, os principais periódicos e eventos 
da área, os autores e instituições mais relevantes e os resíduos e tipos de concreto estudados durante o período de 20 anos na base de dados Web of Science. Possibilitando o norteamento dos estudos nesta área, assim como subsidiar pesquisas que visem difundir a utilização de resíduos no concreto.

\subsection{Concreto com reaproveitamento de resíduos}

O concreto como um material de construção é responsável por severos impactos ambientais. Primeiramente devido ao elevado consumo de matéria-prima natural e, segundo, pelo fato de que ao fim de sua vida útil, o concreto se tornará resíduo de construção e demolição (Wang et al., 2017), de modo que, numa análise de ciclo de vida do material, o resultado é o aumento da geração de resíduo. No entanto, uma parcela significativa desses resíduos gerados pode ser reciclada e utilizada dentro da própria cadeia construtiva que o gerou.

Os resíduos podem ser utilizados no concreto em substituição ao cimento ou agregados. A substituição de cimento por resíduos possui grande apelo ambiental devido à redução na utilização do clínquer, um material com elevado potencial poluidor, e pela utilização de resíduos que de outra maneira seriam destinados a aterros ou locais inadequados (Tavakoli; Hashempour; Heidari, 2018).

Alguns resíduos possuem propriedades pozolânicas (Vishwakarma; Ramachandran, 2018), estes materiais são ricos em sílica e alumina, que reagem com hidróxido de cálcio e água em temperatura ambiente, formando compostos com características cimentíceas (Malhotra; Mehta, 1996). Além de melhorar as propriedades de resistência e àquelas relacionadas com durabilidade do concreto, a substituição de cimento por materiais pozolânicos reduz a emissão de gases responsáveis pelo efeito estufa, como o dióxido de carbono (Tavakoli; Hashempour; Heidari, 2018; Vishwakarma; Ramachandran, 2018).

Os agregados ocupam cerca de 70 a $80 \%$ do volume de concreto e para evitar o grande consumo de matérias-primas naturais já escassas, materiais alternativos como resíduos e subprodutos industriais vêm sendo estudados para substituição dos agregados naturais (Tiwari; Singh; Nagar, 2016; Tavakoli; Hashempour; Heidari, 2018). Os resíduos utilizados em substituição aos agregados devem satisfazer os requisitos mecânicos, reológicos e de durabilidade, para produzir um concreto de boa qualidade, forte e durável (Tiwari; Singh; Nagar, 2016).

A necessidade de adotar metodologias sustentáveis na construção civil é inevitável, 
isto não significa apenas reduzir a emissão de carbono, como também conservar os recursos naturais cruciais ao desenvolvimento da sociedade. Vários pesquisadores adotaram abordagens diferentes para redução na utilização de recursos naturais, mas evitar $100 \%$ da utilização de material prima natural é uma meta praticamente impossível, portanto é necessário considerar a sustentabilidade ao planejar diferentes projetos (Akhtar; Sarmah, 2018).

\subsection{Bibliometria}

A bibliometria atua como uma ferramenta que permite ao pesquisador observar o estado da ciência e tecnologia através da análise da produção global de literatura científica em certo nível de especialização. Esta observação permite situar um cientista em relação a sua comunidade, uma instituição em relação a um país e este em relação ao resto do mundo. (Okubo, 1997).

A utilização de análises quantitativas e métodos estatísticos possibilita analisar o desenvolvimento das publicações em determinado campo, identificando as tendências de pesquisa e periódicos mais utilizados para divulgação do conhecimento (Ji et al., 2018).

O termo bibliometria surgiu no final da década de 1960 por Pritchard, que a define como a aplicação de métodos estatísticos e matemáticos na análise de obras literárias. (Chueke, Amatucci, 2015)

Os avanços da internet e do acesso da informação em geral, aliados ao crescimento das produções científicas permitiram a criação de bases de dados, que funcionam como suportes informacionais, contendo artigos e trabalhos científicos especializados nas diversas áreas do conhecimento, estas bases possuem credibilidade científica e oferecem mecanismos de busca para os materiais bibliográficos (Pizzani et al., 2012).

Lopes et al. (2012) informa que as bases de dados mais utilizadas do mundo são: Web of Science (WoS), Scopus e Google Scholar Metrics. As diferentes bases de dados utilizam métricas distintas e cobrem diferentes dados. O uso de mais de uma base de dados simultaneamente pode ocasionar em diferentes resultados de uma mesma publicação ou pesquisador. A WoS é considerada a base de dados mais tradicional do mundo, possuindo maior cobertura temporal se comparada a Scopus e Google Scholar Metrics. (Lopes et al., 2012) 


\section{Metodologia}

Esta pesquisa caracteriza-se pela abordagem descritiva, do tipo bibliográfico, com levantamento de aspectos quantitativo, objetivando a análise estatística para os dados coletados referentes à produção cientifica sobre concreto com aproveitamento de resíduos. A coleta de dados baseou-se em levantamento bibliométrico das publicações sobre concreto com reaproveitamento de resíduos no acervo internacional da Web of Science, utilizando todas as bases de dados do acervo. O tempo estipulado de pesquisa foi de 20 anos, durante o intervalo entre 1998 a 2017.

O período de coleta de dados é um ponto crítico no estudo bibliométrico, pois muitos autores acreditam que 5 anos são suficientes. Outros estudos sugerem o período mínimo de 10 anos, porém, recomenda-se a análise de 15 ou 20 anos de publicação sobre um tema (Chueke; Amatucci, 2015).

Como critério de seleção de dados o aporte utilizado para as palavras-chave foi: concrete AND (residue OR waste OR tailing). Foram utilizados termos em Inglês para incluir resultados de periódicos internacionais, o operador "and" é utilizado para buscar apenas pelos termos combinados, enquanto que o termo "OR" busca por um dos termos ou por ambos. Após a busca inicial, foram filtrados os resultados por tipo de documento "article" e por país de origem "Brasil".

Foi realizada a leitura exploratória dos 635 artigos preliminares para exclusão das publicações que não possuem relação com o tema deste estudo. Como critério de aceitação, foram considerados os artigos que tratavam diretamente de concreto de cimento Portland com aproveitamento de resíduos em seus objetivos e metodologia, sendo experimental ou não.

Após o refinamento dos artigos, os resultados foram exportados para o software Endnote X9, ferramenta que permite gerenciar os artigos por autor, ano, título ou periódico e verificar resultados duplicados. O software Microfoft Excel 2016 foi utilizado para criação de tabelas e gráficos, a rede de coautorias foi desenvolvida no software VosViewer 1.6.11, o mapa de distribuições geográficas foi criado no software Qgis Desktop 3.6.3 e a nuvem de palavras foi desenvolvida no site Wordclouds (https://www.wordclouds.com/).

\section{Resultados e Discussões}

A filtragem dos artigos resultou em 156 publicações brasileiras sobre concreto com aproveitamento de resíduos na base de dados WoS. A Figura 1 apresenta a evolução das 
publicações brasileiras sobre concreto com reaproveitamento de resíduos no período de 1998 a 2017, apesar de não ter sido contínuo, nota-se um crescimento geral nas publicações durante os anos, com destaque para 2012 (23 artigos), 2014 (24 artigos) e 2017 (28 artigos). Apesar da pesquisa contemplar o período de 1998 a 2017, não foram encontradas publicações brasileiras referentes ao tema analisado durante os cinco primeiros anos do período.

A primeira publicação sobre o tema ocorreu no ano de 2003, com o artigo "Effectiveness of cement-based systems for stabilization and solidification of spent pot liner inorganic fraction" no periódico internacional "Journal of Hazardous Materials". Os autores deste artigo foram: B.I. Silveira, A.E.M. Dantas, J.E.M. Blasques e R.K.P. Santos, todos filiados a Universidade Federal do Pará - UFPA.

Figura 1 - Evolução das publicações brasileiras sobre concreto com reaproveitamento de resíduos.

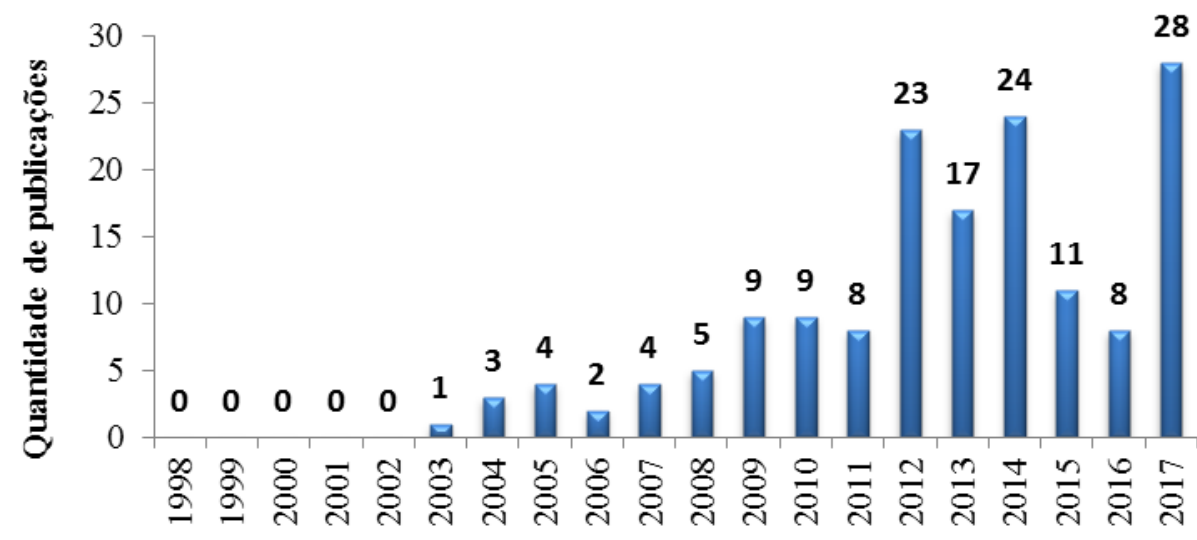

Ano de publicação

Fonte: Autores (2020).

A Tabela 1 ilustra os 10 resíduos com maior frequência nas publicações brasileiras sobre concreto com reaproveitamento de resíduos na base de dados WoS entre 1998 a 2017. O RCD foi o resíduo mais analisado, estando presente em 16,67\% das publicações, em segundo o resíduo de concreto, presente em $8,97 \%$ dos artigos e em terceiro a borracha de pneus, presente em $8,33 \%$ das publicações.

Neste trabalho considerou-se separadamente o resíduo de concreto, resíduo de argamassa e o resíduo cerâmico do RCD, que apresenta composição mista destes materiais, apesar disso, três destes resíduos aparecem entre os 10 mais publicados, com o RCD 
Research, Society and Development, v. 9, n. 9, e260996114, 2020

(CC BY 4.0) | ISSN 2525-3409 | DOI: http://dx.doi.org/10.33448/rsd-v9i9.6114

possuindo o dobro de publicações da borracha de pneus, terceiro resíduo mais publicado no tema de reaproveitamento no concreto.

Tabela 1 - Os 10 resíduos mais frequentes em publicações brasileiras sobre concreto com reaproveitamento de resíduos.

\begin{tabular}{lcc}
\hline \multicolumn{1}{c}{ RESídUOS } & ARTIGOS & $\%$ \\
\hline Resíduo de construção e demolição (RCD) & 26 & 16,67 \\
Resíduo de Concreto & 14 & 8,97 \\
Borracha de pneus & 13 & 8,33 \\
Cinza da Casca de Arroz (CCA) & 8 & 5,13 \\
Cinza do bagaço da cana de açúcar & 8 & 5,13 \\
Lodo de ETA & 8 & 5,13 \\
Resíduo Cerâmico & 8 & 5,13 \\
Resíduo de EVA & 7 & 4,49 \\
Lama vermelha & 6 & 3,85 \\
PET reciclado & 6 & 3,85 \\
\hline
\end{tabular}

Fonte: Autores (2020).

A Figura 2 permite verificar a quantidade de artigos publicados por tipo de concreto. As 156 publicações utilizaram 8 tipos de concreto diferentes, existe predomínio das publicações que utilizam apenas um tipo de concreto $(98,07 \%)$ e apenas três publicações utilizando dois tipos de concreto diferentes. O concreto convencional liderou as publicações, estando presente em $83,97 \%$ dos artigos, seguido por concreto leve $(8,94 \%)$ e concreto auto adensável, presente em 3,20\% dos artigos. 
Research, Society and Development, v. 9, n. 9, e260996114, 2020

(CC BY 4.0) | ISSN 2525-3409 | DOI: http://dx.doi.org/10.33448/rsd-v9i9.6114

Figura 2 - Tipos de concretos mais produzidos nas publicações sobre reaproveitamento de resíduos.

\section{Número de publicações

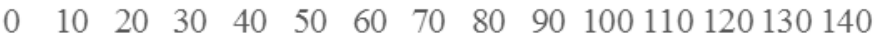

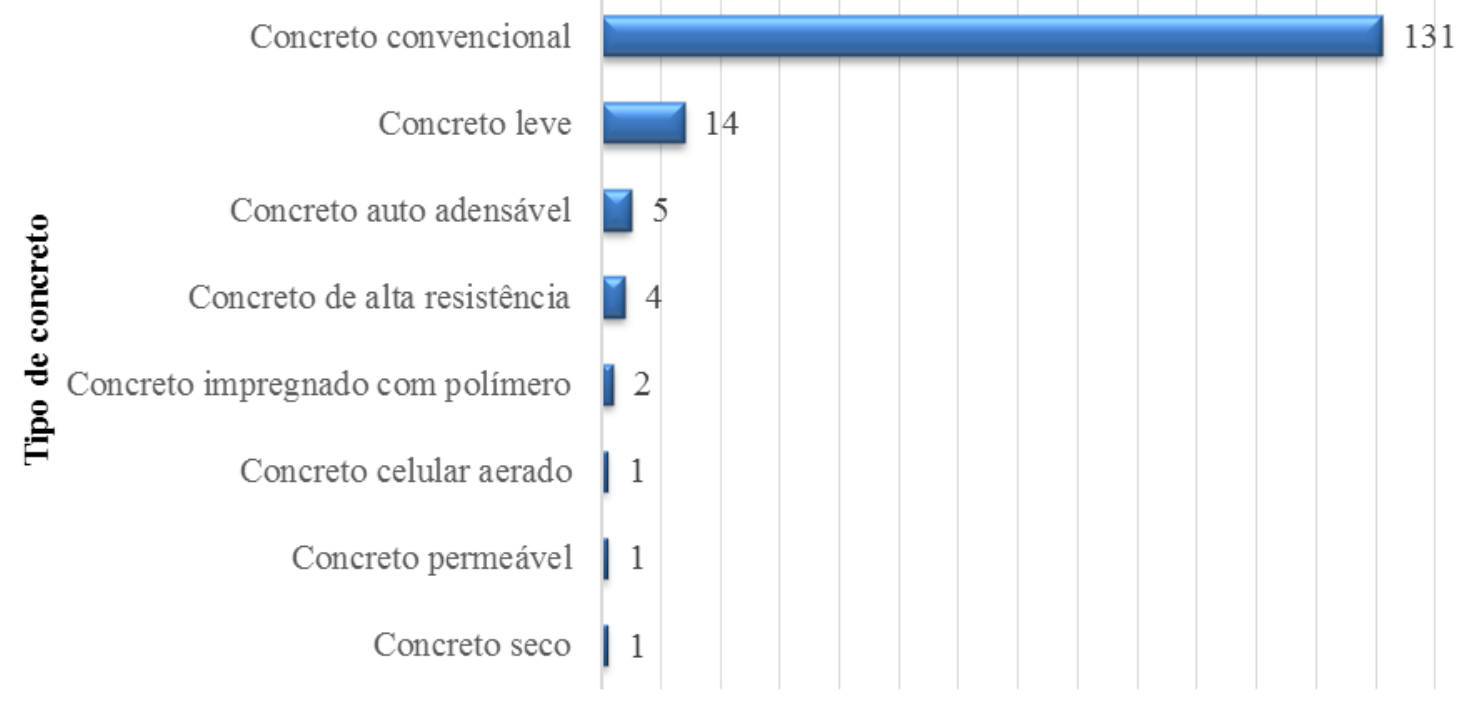

Fonte: Autores (2020).

Dentre os artigos selecionados, 135 foram apresentados em periódicos e 24 em eventos. A soma ultrapassa 156 pois 3 artigos foram publicados tanto em periódicos quanto em eventos. Os 135 artigos estão distribuídos em 38 periódicos, sendo dezenove periódicos com apenas 1 artigo sobre o tema e oito periódicos com 2 artigos publicados. A Tabela 2 apresenta os periódicos que publicaram 3 ou mais artigos sobre o tema, representando 64,10\% de todas as publicações.

O periódico com maior número de publicações de autores brasileiros sobre concreto com reaproveitamento de resíduos na WoS no período de 1998 a 2017 foi a revista internacional "Construction and Building Materials" com 22 artigos, representando 14,10\% das publicações. O número de publicações sobre o tema neste periódico é superior à soma de todos os periódicos que publicaram apenas um artigo (19 artigos no total) e também superior a soma dos periódicos que publicaram 2 artigos (16 artigos no total). Os periódicos brasileiros com maior número de publicações sobre o tema foram as revistas "Matéria (Rio de Janeiro) " e "Revista IBRACON de Estruturas e Materiais" com 15 publicações cada, seguidas pela revista "Ambiente Construído" com 14 publicações. 
Research, Society and Development, v. 9, n. 9, e260996114, 2020

(CC BY 4.0) | ISSN 2525-3409 | DOI: http://dx.doi.org/10.33448/rsd-v9i9.6114

Tabela 2 - Periódicos indexados no WoS com publicações sobre concreto com reaproveitamento de resíduos.

\begin{tabular}{|c|c|c|c|c|}
\hline Periódico & Artigos & $\%$ & $\begin{array}{l}\text { Qualis } \\
\text { Capes }^{1}\end{array}$ & $\begin{array}{l}\text { Fator de } \\
\text { Impacto }\end{array}$ \\
\hline Construction and Building Materials & 22 & 14,10 & $\bar{A} 1$ & $3.485^{2}$ \\
\hline Matéria (Rio de Janeiro) & 15 & 9,62 & B2 & $0.340^{2}$ \\
\hline Revista IBRACON de Estruturas e Materiais & 15 & 9,62 & B1 & $0.0899^{3}$ \\
\hline Ambiente Construído & 14 & 8,97 & B1 & $0.1727^{3}$ \\
\hline Cerâmica & 7 & 4,49 & B2 & $0.0826^{3}$ \\
\hline Waste Management & 7 & 4,49 & A1 & $4.723^{2}$ \\
\hline Cement \& Concrete Composites & 5 & 3,21 & A1 & $4.660^{2}$ \\
\hline Materials Research-Ibero American Journal of Materials & 5 & 3,21 & A2 & $1.103^{2}$ \\
\hline Cement and Concrete Research & 4 & 2,56 & A1 & $5.430^{2}$ \\
\hline ACTA Scientiarum Technology & 3 & 1,92 & B1 & $0.231^{2}$ \\
\hline Journal of Cleaner Production & 3 & 1,92 & A1 & $5.651^{2}$ \\
\hline
\end{tabular}

${ }^{1}$ Quadriênio 2013-2016 na área Engenharias I

2 Journal Citation Reports

${ }^{3}$ SciElo

Fonte: Autores (2020).

Foram publicados 24 artigos brasileiros sobre concreto com reaproveitamento de resíduos em 14 eventos nacionais e internacionais indexados no WoS (Tabela 3) durante o período de 1998 a 2017. O evento com maior número de publicações foi o "57TH Brazilian Ceramic Conference CBC" que ocorreu em Natal-RN em 2013 e contou com 5 artigos sobre o tema, representando $3,16 \%$ do total de publicações. Os eventos "13TH International Conference on non-Conventional Materials and Technologies", "20TH Brazilian Conference on Materials Science and Engineering CBECIMAT" e "8TH Latin American Conference on Powder Technology" contaram com 3 publicações cada. Os demais eventos tiveram apenas 1 publicação relacionada ao tema. 
Research, Society and Development, v. 9, n. 9, e260996114, 2020

(CC BY 4.0) | ISSN 2525-3409 | DOI: http://dx.doi.org/10.33448/rsd-v9i9.6114

Tabela 3 - Eventos científicos indexados no WoS com publicações sobre concreto com reaproveitamento de resíduos.

\begin{tabular}{|c|c|c|}
\hline EVENTO & ARTIGOS & $\%$ \\
\hline 57TH Brazilian Ceramic Conference CBC & 5 & 3,16 \\
\hline 13TH International Conference on non-Conventional Materials and Technologies & 3 & 1,90 \\
\hline 20TH Brazilian Conference on Materials Science and Engineering CBECIMAT & 3 & 1,90 \\
\hline 8TH Latin American Conference on Powder Technology & 3 & 1,90 \\
\hline 2ND Global Conference on Civil Structural and Environmental Engineering GCCSEE 2013 & 1 & 0,63 \\
\hline 2ND International Conference on Mechatronics and Control Engineering ICMCE 2013 & 1 & 0,63 \\
\hline 32ND Annual Meeting of the Sociedade Brasileira de Química & 1 & 0,63 \\
\hline $\begin{array}{l}\text { 3RD International Conference on Chemical Engineering and Advanced Materials CEAM } \\
2013\end{array}$ & 1 & 0,63 \\
\hline $\begin{array}{l}\text { 3RD International Conference on Green Building Materials and Civil Engineering } \\
\text { GBMCE } 2013\end{array}$ & 1 & 0,63 \\
\hline 4TH Meeting of the Brazilian Society for Materials Research & 1 & 0,63 \\
\hline $\begin{array}{l}\text { 5TH Kumamoto International Workshop on Fracture Acoustic Emission and } \\
\text { Nondestructive Evaluation in Concrete KIFA } 5\end{array}$ & 1 & 0,63 \\
\hline 6TH International Latin American Conference on Power Technology & 1 & 0,63 \\
\hline Global Stone Congress 2012 & 1 & 0,63 \\
\hline International Conference on Material Engineering and Application ICMEA 2014 & 1 & 0,63 \\
\hline
\end{tabular}

Fonte: Autores (2020).

A Tabela 4 apresenta os autores com 5 ou mais publicações sobre concreto com reaproveitamento de resíduos no WoS durante o período de 1998 a 2017. Esta amostragem de 16 autores representa apenas 3,74\% dos 427 pesquisadores identificados, no entanto, os mesmos participaram da autoria de $64,10 \%$ dos artigos relacionados ao tema, enquanto que 328 autores $(76,81 \%)$ publicaram apenas 1 artigo. 
Research, Society and Development, v. 9, n. 9, e260996114, 2020

(CC BY 4.0) | ISSN 2525-3409 | DOI: http://dx.doi.org/10.33448/rsd-v9i9.6114

Tabela 4 - Autores com frequência de publicação de 5 ou mais artigos sobre concreto com reaproveitamento de resíduos.

\begin{tabular}{lll}
\hline \multicolumn{1}{c}{ Autor } & Artigos & $\%$ \\
\hline Dal Molin, D. C. C. & 9 & $5,77 \%$ \\
Toledo, R. D. & 8 & $5,13 \%$ \\
Leite, M. B. & 7 & $4,49 \%$ \\
Portella, K. F. & 7 & $4,49 \%$ \\
Sales, A. & 7 & $4,49 \%$ \\
Alexandre, J. & 6 & $3,85 \%$ \\
Lintz, R. C. C. & 6 & $3,85 \%$ \\
Monteiro, S. N. & 6 & $3,85 \%$ \\
Morelli, M. R. & 6 & $3,85 \%$ \\
Pepe, M. & 6 & $3,85 \%$ \\
Ribeiro, J. L. D. & 6 & $3,85 \%$ \\
Vieira, C. M. F. & 6 & $3,85 \%$ \\
Cabral, A. E. B. & 5 & $3,21 \%$ \\
Joukoski, A. & 5 & $3,21 \%$ \\
Koenders, E. A. B. & 5 & $3,21 \%$ \\
Schalch, V. & 5 & $3,21 \%$ \\
\hline
\end{tabular}

Fonte: Autores (2020).

A autora Dal Molin, D.C.C, filiada à Universidade Federal do Rio Grande do SulUFRGS é a autora mais produtiva sobre este tema, participando da autoria de 9 artigos, o que representa 5,77\% dos artigos totais. Cabe ressaltar que os artigos analisados possuem entre $1 \mathrm{e}$ 8 autores, todos considerados na coautoria dos artigos (Figura 3).

Os artigos analisados possuem uma média de 3,99 autores por publicação, resultado próximo a média de 3,5 autores encontrado por Cañas-Guerrero et al. (2013) para publicações brasileiras sobre Engenharia civil no WoS durante o período de 1997 a 2011. Observa-se a predominância de artigos com 3 autores (25,64\%), seguido pelas publicações com 4 (24,36\%) e $5(19,87 \%)$ autores respectivamente. 
Research, Society and Development, v. 9, n. 9, e260996114, 2020

(CC BY 4.0) | ISSN 2525-3409 | DOI: http://dx.doi.org/10.33448/rsd-v9i9.6114

Figura 3 - Número de autores por artigo relacionado ao tema de concreto com reaproveitamento de resíduos.

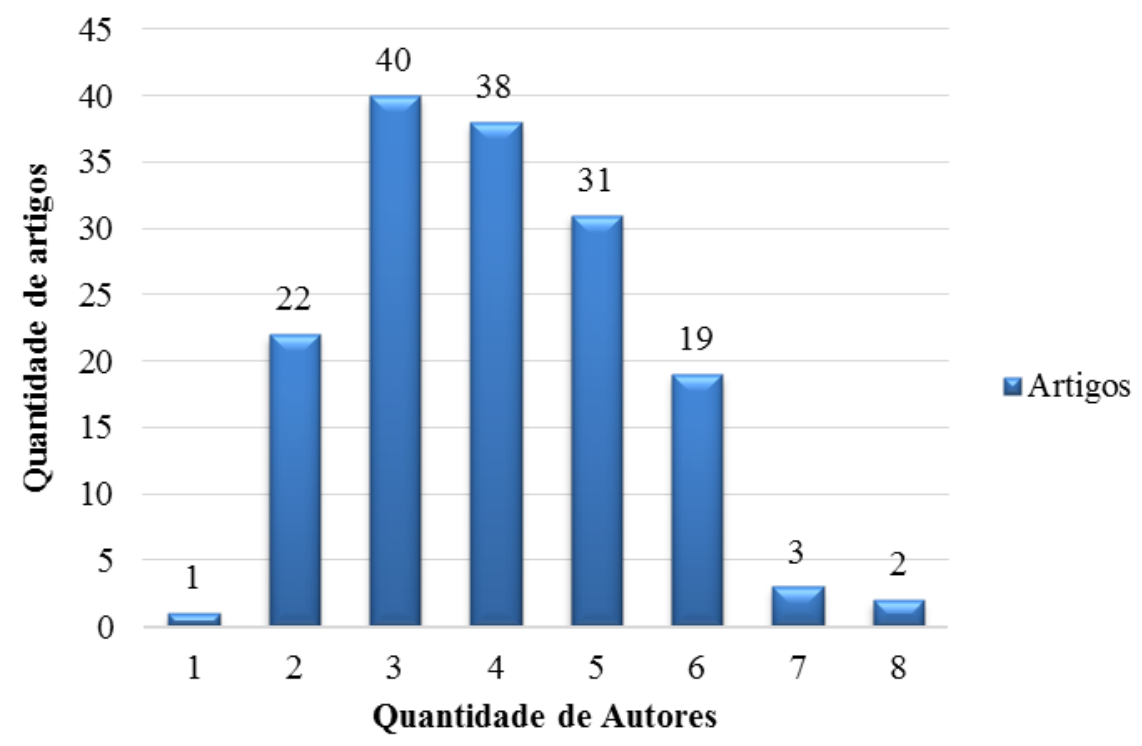

Fonte: Autores (2020).

A Figura 4 apresenta o mapeamento da rede de coautorias dos artigos sobre concreto com reaproveitamento de resíduos. Foram verificados 70 grupos de autores, variando entre 2 e 18 membros. Machado, Helleno e Simon (2016) afirmam que a rede de coautorias mostra a conexão entre autores relacionados, dividindo-os em grupos denominados Clusters, cada Cluster agrega os itens similares e possui uma cor diferente. A dimensão dos círculos representa a quantidade de publicações de cada autor.

Os Clusters com mais ligações aparecem em destaque no mapa, sendo o maior grupo centralizado por Portella, K. F., com 18 autores, seguido pelos Clusters centralizados por Lintz, R. C. C. e Toledo, R. D. com 17 membros cada. Apesar de Dal Molin, D.C.C ser a autora mais produtiva, o Cluster a qual ela pertence é o sexto maior, com 14 membros, o que sugere que a mesma concentra as publicações com os mesmos autores relacionados. 
Research, Society and Development, v. 9, n. 9, e260996114, 2020

(CC BY 4.0) | ISSN 2525-3409 | DOI: http://dx.doi.org/10.33448/rsd-v9i9.6114

Figura 4 - Rede de coautoria dos artigos sobre concreto com reaproveitamento de resíduos.

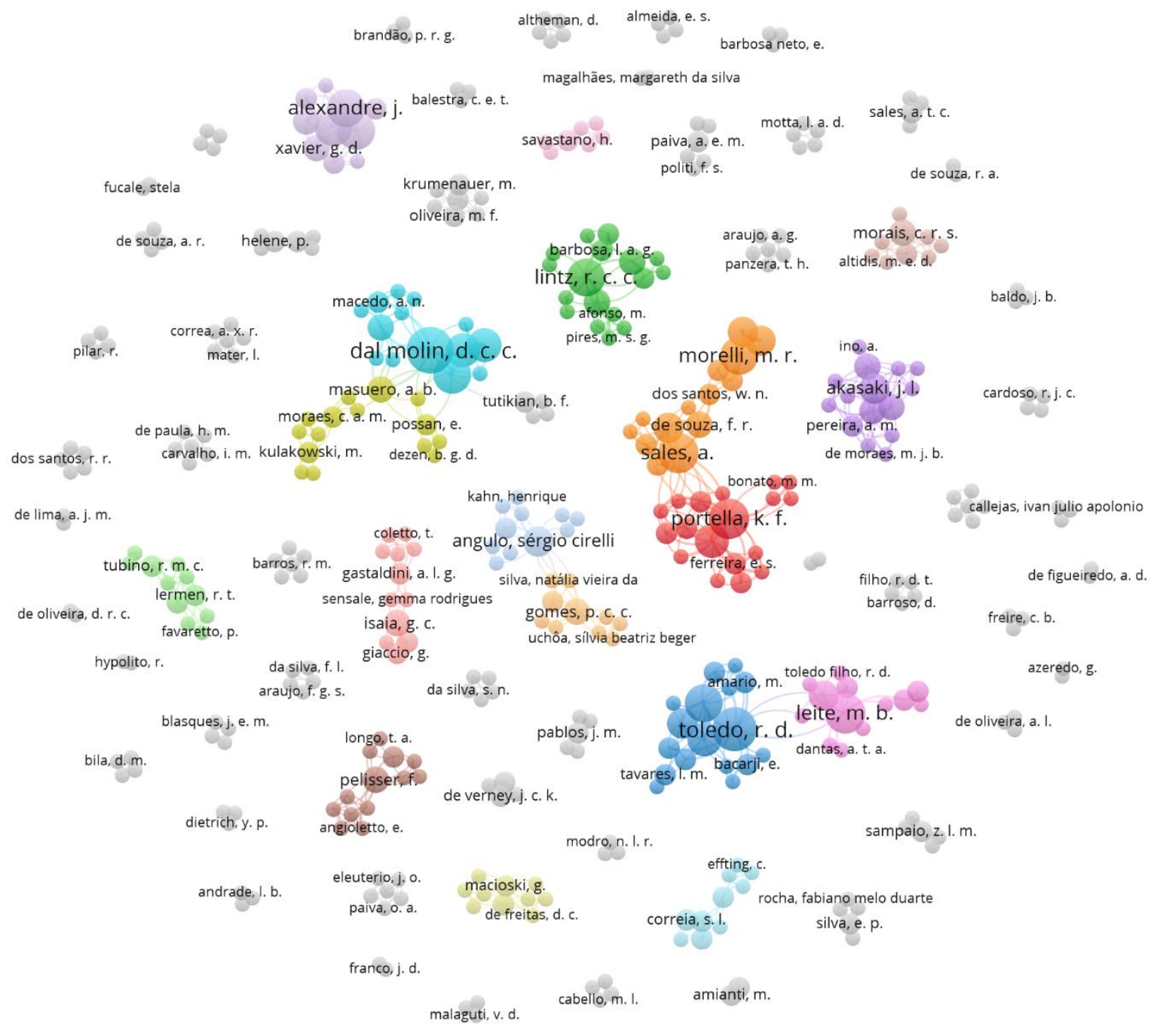

Fonte: Autores (2020).

A rede analisada demonstrou baixo grau de conexões, com 50 Clusters com 6 ou menos membros, tal fato pode ser influenciado pela heterogeneidade do tema ou pelo número de artigos analisados.

Os 156 artigos foram publicados por autores de 104 filiações, a Figura 5 demonstra as 23 instituições com 5 ou mais publicações sobre concreto com reaproveitamento de resíduos no WoS durante o período de 1998 a 2017. Soares et al. (2016) destacam que um artigo pode ser contado mais de uma vez caso os autores pertençam a filiações diferentes.

Entre as 104 filiações, 22 são instituições estrangeiras (aproximadamente 21\%), sendo que três possuem mais de 4 publicações: University of Salerno - UNISA (Itália), 
Universidade de Aveiro - UA (Portugal) e Delft University of Technology - TU Delft (Holanda).

A Universidade de São Paulo - USP se destacou como a instituição mais produtiva, concentrando $14 \%$ das publicações. Esta posição da USP é semelhante a encontrada por Soares et al. (2016) sobre publicações brasileiras no tema de construção e tecnologia da construção. Entre as 20 instituições nacionais mais produtivas, oito estão situadas na região sudeste, oito na região sul, três na região nordeste e uma na região norte.

Figura 5 - Instituições com 4 ou mais publicações sobre concreto com reaproveitamento de resíduos.

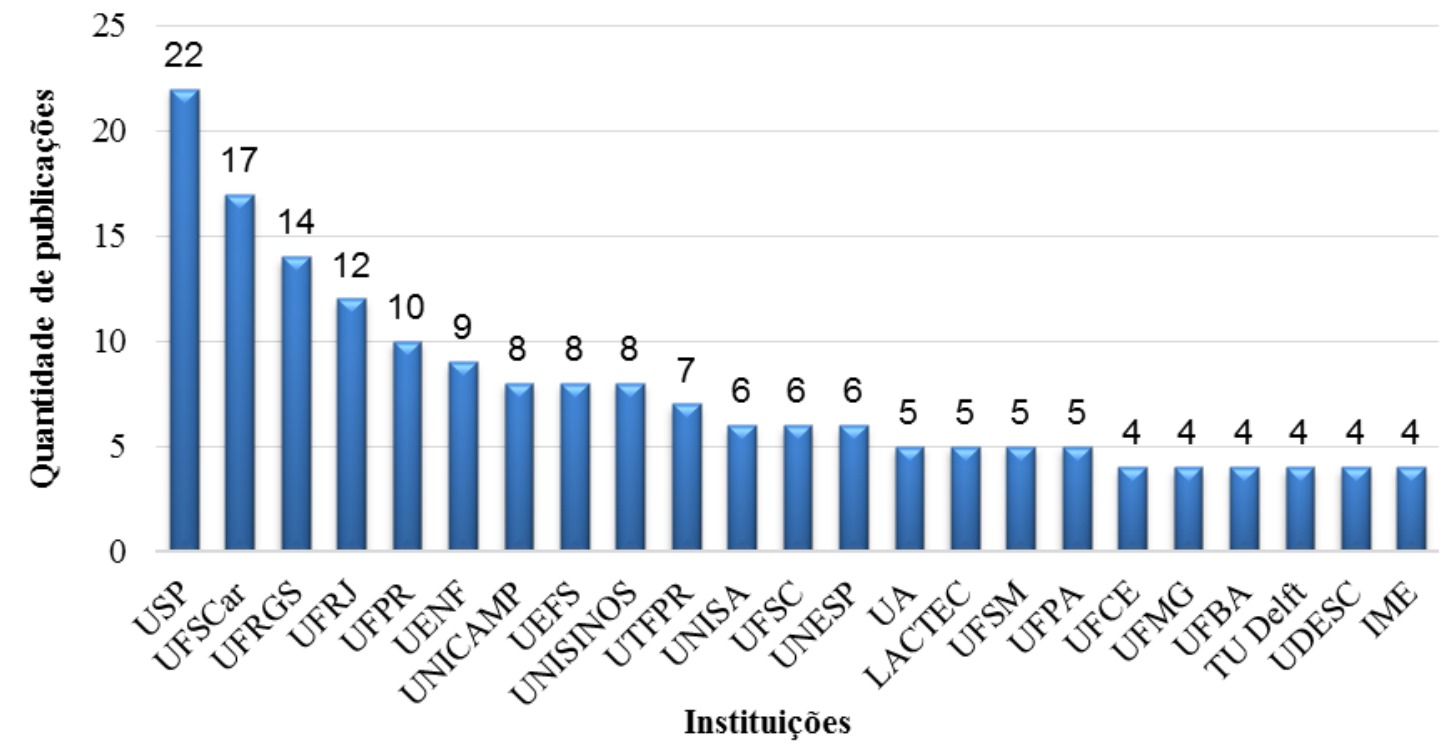

Fonte: Autores (2020).

Foram publicados 33 artigos em coautoria com autores estrangeiros, pertencentes a instituições da Itália (seis artigos), Portugal (seis artigos), Holanda (quatro artigos), Argentina (três artigos), Estados Unidos (três artigos), Espanha (três artigos), Alemanha (dois artigos), Austrália (dois artigos), Colômbia (dois artigos), Inglaterra (um artigo) e Uruguai (um artigo). A Figura 6 representa o mapa de distribuição geográfica, destacando os países com publicações em coautoria com autores brasileiros e o número de publicações por estado brasileiro.

É possível verificar a preferência por coautores da Europa, com 57,57\% das publicações. Apenas dois artigos (6,06\%) foram publicados fora do eixo Europa-América. 
Nenhum autor da Ásia ou África publicou artigo em coautoria com autores brasileiros neste tema.

Figura 6 - Mapa de distribuição geográfica das publicações brasileiras sobre concreto com reaproveitamento de resíduos.

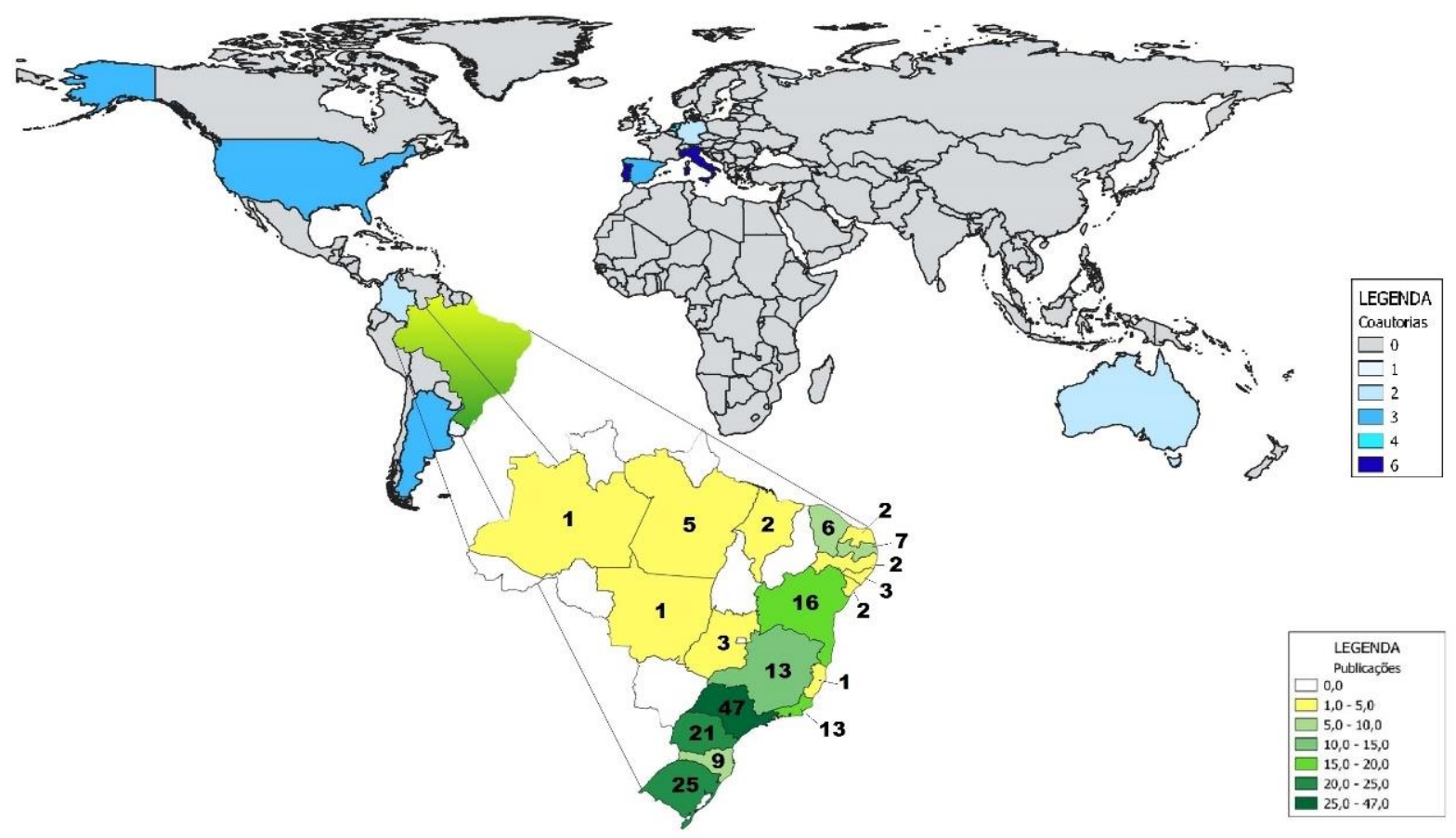

Fonte: Autores (2020).

A maior concentração de publicações brasileiras sobre concreto com reaproveitamento de resíduos está localizada na região Sudeste, com autoria de 57,05\% das publicações, seguido pela região Sul $(35,26 \%)$, Nordeste $(25,64 \%)$, Norte $(3,85 \%)$ e CentroOeste (2,56\%). Quanto as unidades da federação, São Paulo foi o estado brasileiro mais produtivo (30,13\%), seguido pelo Rio Grande do Sul (16,03\%) e Paraná (13,56\%), enquanto que oito estados não apresentaram publicações sobre o tema.

A prevalência das publicações do estado de São Paulo, assim como da região sudeste, sul e nordeste corroboram com o resultado de Soares et al. (2016) sobre a produção brasileira sobre tecnologia de construção e edificações.

Os 156 artigos selecionados foram citados 1566 vezes durante o período de 2005 a outubro de 2018 por 1366 artigos, uma média de 10,03 citações por artigo. Os 10 artigos mais citados estão organizados na Tabela 5. Apesar desta amostragem representar apenas $6,41 \%$ da amostra de artigos eles representam 44,51\% do total de citações recebidas. Ainda que estes 
Research, Society and Development, v. 9, n. 9, e260996114, 2020

(CC BY 4.0) | ISSN 2525-3409 | DOI: http://dx.doi.org/10.33448/rsd-v9i9.6114

artigos tenham se referidoa autores brasileiros, nenhuma destas publicações foi realizada em periódicos nacionais.

$\mathrm{O}$ artigo "Durability of recycled aggregates concrete: a safe way to sustainable development" que pesquisou a utilização de RCD na dosagem de concreto foi o artigo mais relevante, sendo citado 157 vezes, o equivalente a 10,03\% do total de citações. Este resultado está de acordo com a verificação de que RCD foi o resíduo mais publicado por autores nacionais, porém o terceiro artigo mais citado se refere ao "resíduo do processo de britagem e lixiviação de escória de alumínio secundária”, que recebeu 79 citações, no entanto, este possui apenas uma única publicação por autores brasileiros durante o período na WoS, o que indica que possivelmente é um tema mais recorrente em outros países.

Tabela 5 - As 10 publicações mais influentes sobre concreto com reaproveitamento de resíduos.

\begin{tabular}{|c|c|c|}
\hline TÍTULO DO ARTIGO & CITAÇÕES & $\%$ \\
\hline Durability of recycled aggregates concrete: a safe way to sustainable development & 157 & 10,03 \\
\hline $\begin{array}{l}\text { Concrete made with recycled tire rubber: Effect of alkaline activation and silica fume } \\
\text { addition }\end{array}$ & 100 & 6,39 \\
\hline $\begin{array}{l}\text { Solid waste from aluminum recycling process: characterization and reuse of its economically } \\
\text { valuable constituents }\end{array}$ & 79 & 5,04 \\
\hline Mechanical properties modeling of recycled aggregate concrete & 60 & 3,83 \\
\hline Use of Brazilian sugarcane bagasse ash in concrete as sand replacement & 59 & 3,77 \\
\hline Evaluation of concrete incorporating bottom ash as a natural aggregates replacement & 57 & 3,64 \\
\hline Concrete incorporating rice-husk ash without processing & 56 & 3,58 \\
\hline Modeling of mechanical properties and durability of recycled aggregate concretes & 45 & 2,87 \\
\hline Compressive stress-strain behavior of steel fiber reinforced-recycled aggregate concrete & 43 & 2,75 \\
\hline Copper slag waste as a supplementary cementing material to concrete & 41 & 2,62 \\
\hline
\end{tabular}

Fonte: Autores (2020).

Foi realizada a análise das palavras-chave dos artigos do acervo da WoS, com 322 palavras-chave diferentes, as 20 palavras-chave com 6 ou mais repetições estão dispostas na 
Research, Society and Development, v. 9, n. 9, e260996114, 2020

(CC BY 4.0) | ISSN 2525-3409 | DOI: http://dx.doi.org/10.33448/rsd-v9i9.6114

Tabela 6. Para esta análise considerou-se apenas as palavras-chave do abstract dos artigos, sendo que apenas um artigo não apresentou abstract.

Tabela 6 - As 20 palavras-chave mais frequentes nas publicações sobre concreto com reaproveitamento de resíduos.

\begin{tabular}{|c|c|c|c|}
\hline Palavras chave & Frequência & Palavras chave & Frequência \\
\hline Concrete & 59 & $\begin{array}{l}\text { Recycled aggregate } \\
\text { concrete }\end{array}$ & 8 \\
\hline $\begin{array}{l}\text { Construction and } \\
\text { demolition waste }\end{array}$ & 18 & Alternative materials & 7 \\
\hline Compressive strength & 17 & Mortar & 7 \\
\hline Recycled aggregate & 14 & Construction & 7 \\
\hline Recycling & 14 & Aggregate & 6 \\
\hline Waste & 10 & Construction materials & 6 \\
\hline Lightweight concrete & 9 & Portland cement & 6 \\
\hline Durability & 8 & Rice husk ash & 6 \\
\hline Mechanical properties & 8 & $\begin{array}{l}\text { Sugar cane bagasse } \\
\text { ash }\end{array}$ & 6 \\
\hline Rubber tire & 8 & $\begin{array}{l}\text { Water treatment } \\
\text { sludge }\end{array}$ & 6 \\
\hline
\end{tabular}

Fonte: Autores (2020).

A palavra-chave mais utilizada foi "Concrete", presente diretamente em 59 artigos, porém entre as 20 palavras-chave mais utilizadas, ela aparece indiretamente em "Lightweight concrete" com 9 repetições e em "Recycled aggregate concrete" com 8 repetições. O mesmo ocorre com o termo "Waste", que aparece como a sexta palavra mais utilizada, com 10 repetições diretas, porém aparece indiretamente em "Construction and demolition waste", presente em 18 artigos.

De acordo com Francisco (2011), ao considerar a frequência de palavras isoladas, é possível fortalecer a ocorrência de palavras provenientes de termos compostos. Para esta 
análise, foi considerada a metodologia de Francisco (2011), utilizando as 100 palavras-chave isoladas mais frequentes para criação de uma nuvem de palavras (Figura 7), com destaque as 15 palavras-chave isoladas mais utilizadas.

Figura 7 - Nuvem de palavras-chave isoladas nas publicações sobre concreto com reaproveitamento de resíduos.

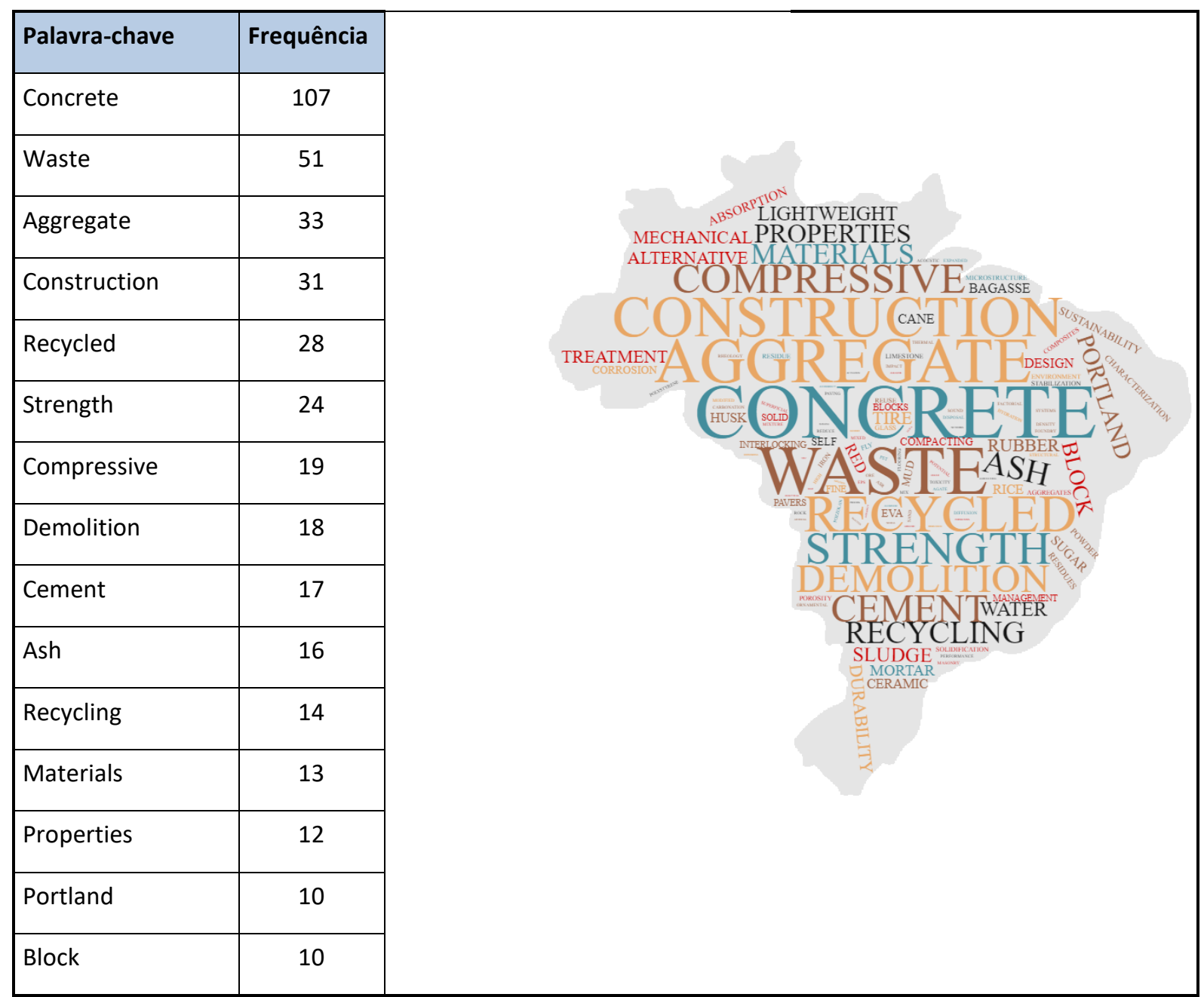

Fonte: Autores (2020).

Ao considerar apenas as palavras isoladas, "Concrete" continuar a ser a palavra mais utilizada, porém sua frequência aumentou de 59 para 107 utilizações, enquanto que "Waste" passou de sexta palavra mais frequente para segunda posição, aumentando de 10 para 51 repetições.

Os 156 artigos foram publicados em três idiomas, apesar de possuir predominantemente autores brasileiros, o idioma mais utilizado nas publicações foi o inglês, com 108 artigos publicados, seguido por 46 publicações em português e 2 em espanhol. A Figura 8 demonstra o percentual de publicações em cada idioma. 
Figura 8 - Idioma das publicações sobre concreto com reaproveitamento de resíduos.

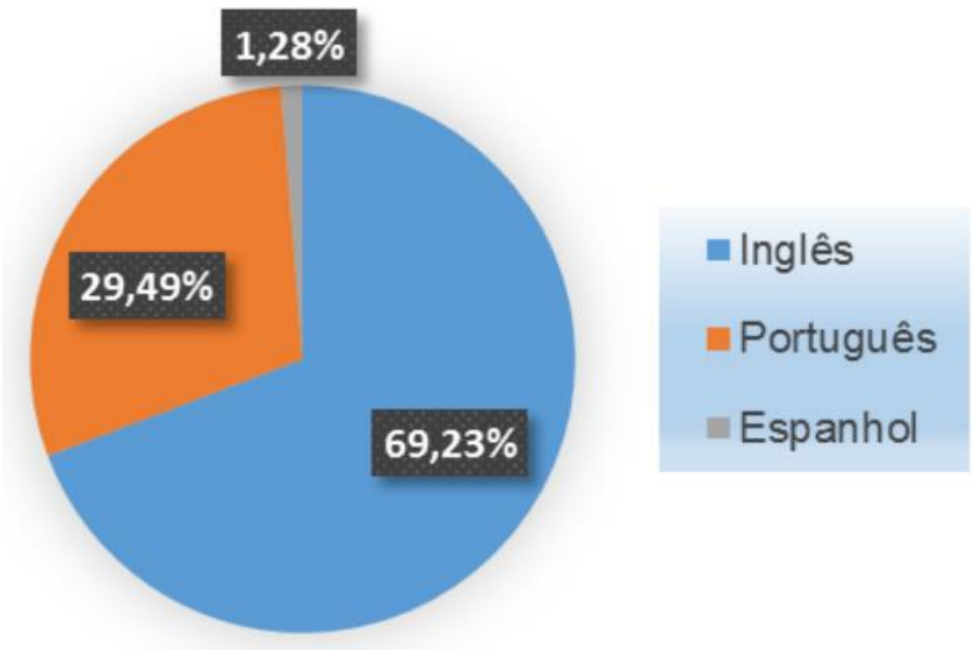

Fonte: Autores (2020).

Nota-se que quase $70 \%$ das publicações acerca deste tema foram na lingua inglesa, isso se deve a sua utilização com linguagem universal, inclusive no meio científico.

\section{Considerações Finais}

A utilização de ferramentas bibliométricas possibilitou divulgar uma visão geral sobre o estado atual das publicações nacionais sobre concreto com reaproveitamento de resíduos, apresentando informações relacionadas a evolução das publicações, resíduos e tipos de concreto mais frequentes, periódicos e eventos que divulgaram o tema, produtividade e influência de autores e instituições, palavras-chave mais recorrentes e idioma das publicações.

A pesquisa considerou o período de 20 anos, compreendendo entre 1998 a 2017, sendo possível identificar um crescimento geral das publicações, com destaque para a produtividade dos anos de 2012, 2014 e 2017, que individualmente obtiveram mais publicações do que a soma dos 10 primeiros anos analisados.

Apesar do primeiro artigo sobre o tema neste período ter sido publicado na Universidade Federal do Pará, as maiores concentrações de publicações pertencem as regiões sudeste e sul, respectivamente, com destaque a Universidade de São Paulo, Universidade Federal de São Carlos e Universidade Federal do Rio Grande do Sul. Quanto às coautorias internacionais, a preferência foi para autores da Europa e Américas.

O resíduo de construção e demolição foi o resíduo com maior número de publicações e foi protagonista do artigo mais influente durante o período, sendo também a segunda 
palavra chave mais utilizada nos artigos (Construction and demolition waste), perdendo apenas para concreto (concrete).

Apesar da construção civil ser o tema de muitos trabalhos, as publicações brasileiras sobre concreto com reaproveitamento de resíduos ainda são incipientes, foram verificadas lacunas de publicações, como o baixo grau de colaboração entre autores da área, principalmente no âmbito internacional, com autores da Ásia e África, além da baixa frequência de publicações de trabalhos que utilizem resíduos na produção de concretos especiais.

Devido ao elevado consumo de recursos naturais e geração de resíduos ocasionados pela construção civil, o reaproveitamento de resíduos no concreto tem demonstrado grande potencial de sustentabilidade, ao permitir que resíduos com pouca utilidade sejam aproveitados, evitando assim, a extração de matérias primas naturais, além de possibilitar o reaproveitamento do próprio concreto, que pode se tornar resíduo, seja pela ação do homem, ou até mesmo, devido a um desastre natural.

Recomenda-se que sejam realizados estudos viabilizando o uso da incorporação de determinados resíduos que foram constatados satisfatórios nos ensaios dos trabalhos aqui elencados. Principalmente, analisando a viabilidade técnica econômica do seu uso em determinadas regiões.

\section{Referências}

ABRELPE. (2017). Panorama dos resíduos sólidos no brasil, Associação Brasileira das Empresas de Limpeza Pública e Resíduos Especiais.

Akhtar, Ali, Sarmah, Ajit K. (2018). Construction and demolition waste generation and properties of recycled aggregate concrete: A global perspective. Journal Of Cleaner Production. [s.1.], 186, 262-281. http://dx.doi.org/10.1016/j.jclepro.2018.03.085.

Brasileiro, L. L., Matos, J. M. E. (2015). Revisão bibliográfica: reutilização de resíduos da construção e demolição na indústria da construção civil. Cerâmica, [s.1.], 61(358), 178-189. FapUNIFESP (SciELO). http://dx.doi.org/10.1590/0366-69132015613581860.

Cabral, A. E. B. (2007). Modelagem de propriedades mecânicas e de durabilidade de concretos produzidos com agregados reciclados, considerando-se a variabilidade da 
composição do RCD. Escola de Engenharia de São Carlos, Universidade de São Paulo, São Carlos (Tese de doutorado).

Cañas-Guerrero, I. et al. (2013). Analysis of research activity in the field "Engineering, Civil" through bibliometric methods. Engineering Structures, [s.1.], 56, 2273-2286. Elsevier BV. http://dx.doi.org/10.1016/j.engstruct.2013.08.038

Chueke, G. V., Amatucci, M. (2015). O que é bibliometria? Uma introdução ao Fórum. Internext, [s.1.], 10(2), 1-5. http://dx.doi.org/10.18568/1980-4865.1021-5

Francisco, E. R. (2011). RAE-eletrônica: exploração do acervo à luz da bibliometria, geoanálise e redes sociais. Revista de Administração de Empresas, [s.1.], 51(3), 280-306. FapUNIFESP (SciELO). http://dx.doi.org/10.1590/s0034-75902011000300008

Ji, Ling et al. (2018). The evolution of Resources Conservation and Recycling over the past 30 years: A bibliometric overview. Resources, Conservation and Recycling, [s.1.], 134, 34-43. Elsevier BV. http://dx.doi.org/10.1016/j.resconrec.2018.03.005.

Lopes, S. et al. (2012). A bibliometria e a avaliação da produção científica: indicadores e ferramentas. Actas dos Congressos Nacionais de Bibliotecários, Arquivistas e Documentalistas, (11), 1-7.

Machado, R. H. C.; Helleno, A. L.; Simon, A. T. (2016). Estudo bibliométrico da produção científica internacional sobre DES aplicado à logística. Revista de Ciência \& Tecnologia, 19(38), 17-31.

Malhotra, V. M, \& Mehta, P. K. (1996). Pozzolanic and cementitious materials. Advances in concrete technology. Canadá: Taylor \& Francis, Vol 1.

Morais, M. H. L., Souza, W. M., \& Ribeiro, A. J. A.(2020). Use of red ceramic waste as fine aggregate in concretes. Research, Society and Development, 9(7), 1-17, e357974145. 
Moura, W. A., Leite, M. B., \& Bastos, A. J. O. (2013). Avaliação do uso de resíduo de serragem de pedra Cariri (RSPC) para produção de concretos convencionais. Ambiente Construído, [s.1.], 13(1), 07-24. http://dx.doi.org/10.1590/s1678-86212013000100002.

Okubo, Y. (1997). Bibliometric Indicators and Analysis of Research Systems. Oecd Science, Technology And Industry Working Papers, [s.1.], 1-70. Organisation for Economic CoOperation and Development (OECD). http://dx.doi.org/10.1787/208277770603.

Pizzani, L., et al. (2012). A arte da pesquisa bibliográfica na busca do conhecimento. Rdbci: Revista Digital de Biblioteconomia e Ciência da Informação, [s.1.], 10(1), 53-66. Universidade Estadual de Campinas. http://dx.doi.org/10.20396/rdbci.v10i1.1896.

Silva, N. G. (2006). Argamassa de revestimento de cimento, cal e areia britada de rocha calcária. Mestrado em Construção civil, Universidade Federal do Paraná, Curitiba, 180 f. (Dissertação de mestrado).

Soares, P. B., et al. (2016). Análise bibliométrica da produção científica brasileira sobre Tecnologia de Construção e Edificações na base de dados Web of Science. Ambiente Construído, [s.1.], 16(1), 175-185. http://dx.doi.org/10.1590/s1678-86212016000100067.

Vishwakarma, V., \& Ramachandran, D. (2018). Green Concrete mix using solid waste and nanoparticles as alternatives - A review. Construction And Building Materials, [s.1.], 162, 96103. Elsevier BV. http://dx.doi.org/10.1016/j.conbuildmat.2017.11.174.

Tavakoli, D., Hashempour, M., \& Heidari, A. (2018). Use of Waste Materials in Concrete: A review. Pertanika J. Sci. \& Technol., [s. L.], 2(26), 499-522.

Tiwari, A., Singh, S., \& Nagar, R. (2016). Feasibility assessment for partial replacement of fine aggregate to attain cleaner production perspective in concrete: A review. Journal Of Cleaner Production, [s.1.], 135, 490-507. http://dx.doi.org/10.1016/j.jclepro.2016.06.130.

Wang, L., et al. (2017). An environmentally friendly method to improve the quality of recycled concrete aggregates. Construction and Building Materials, [s.1.], 144, 432-441. Elsevier BV. http://dx.doi.org/10.1016/j.conbuildmat.2017.03.191. 
Research, Society and Development, v. 9, n. 9, e260996114, 2020

(CC BY 4.0) | ISSN 2525-3409 | DOI: http://dx.doi.org/10.33448/rsd-v9i9.6114

Porcentagem de contribuição de cada autor no manuscrito

Felipe José Marques Mesquita - 30\%

Taiana da Silva Ferreira - 20\%

Geilma Lima Vieira - 15\%

Heleno Fülber - $15 \%$

Júnior Hiroyuki Ishihara - 20\% 